Communications in Physics, Vol. 24, No. 4 (2014), pp. 371-380

DOI:10.15625/0868-3166/24/4/5241

\title{
SENSITIVITY LIMIT OF THE VATLY RADIO TELESCOPE: OBSERVING THE MOON
}

\author{
NGUYEN THI PHUONG, NGUYEN THI THAO, PIERRE DARRIULAT, PHAM NGOC DIEP, \\ DO THI HOAI, PHAM THI TUYET NHUNG, AND PHAM TUAN ANH \\ Department of Astrophysics (DAP), Vietnam National Satellite Center (VNSC), \\ Vietnam Academy of Science and Technology (VAST), \\ 18 Hoang Quoc Viet, Cau Giay, Hanoi, Vietnam
}

E-mail: ntphuong02@vnsc.org.vn

Received 10 November 2014

Accepted for publication 22 December 2014

\begin{abstract}
The Moon has been observed using the VATLY radio telescope at frequencies of 1420.4 and $1417.6 \mathrm{MHz}$ in order to study the behaviour of the instrument in a domain of flux density close to the limit of its sensitivity. Drift scans have revealed a Moon flux density of $0.83 \pm 0.16 \mathrm{kJy}$ corresponding to a Moon black body temperature of $207 \pm 40 \mathrm{~K}$. From these results, a limit sensitivity of $\sim 300$ Jy has been inferred in agreement with earlier coarser estimates.
\end{abstract}

\section{INTRODUCTION}

The Moon is known to be a strong radio source. At the limit of the ability of our telescope, it is a convenient target for the study of its sensitivity. Its radio emission has been studied in detail in the third quarter of the past century [1]. At infrared wavelengths there are variations correlated with the lunar phase which are due to solar heating. At centimetre wavelengths such variations are nearly negligible. This is because radio emission (which is thermal) arises from below the surface, underneath the regolith, where the rock is heated by conduction and the variations lag behind solar heating.

Earlier studies of the performance of the VATLY radio telescope [2,3], including observations of the Crab (Taurus A) and measurements of fluctuations of the antenna temperature during quiet nights, have suggested that the limit sensitivity of the instrument would be a few hundred Jansky, mostly limited by man-made radio-frequency interferences (RFI) and gain instabilities caused, in particular, by temperature variations. In order to obtain a more precise, and more reliable evaluation, we have observed the Moon using either a beam-switching technique or drift scans. These observations and their analysis are the subject of the present article.

Details about the VATLY radio telescope and its performance have been published earlier $[2,3]$ and do not need to be repeated here. It is sufficient to recall that it is equipped with a fully steerable parabolic dish, $2.6 \mathrm{~m}$ in diameter, remotely adjustable in elevation and azimuth. 
It is operated at frequencies between 1400 and $1440 \mathrm{MHz}$. The reflected wave is deteced at focus by a helical feed, where it is locally pre-amplified, shifted to lower frequency using standard superheterodyne, amplified and digitized. Standard data collection consists in a sequence of successive measurements of $\sim 8.2 \mathrm{~s}$ duration each, digitized in the form of a frequency histogram covering $\sim 1.2 \mathrm{MHz}$ in 156 bins of $\sim 7.8 \mathrm{kHz}$ each. The angular aperture of the main lobe (the "beam") is well described by a Gaussian having a $\sigma$ of $2.3^{\circ}$ and the pointing accuracy is measured to be $0.22^{\circ}$ in $a \times \cos (h)$ and $0.11^{\circ}$ in $h$ where $a$ and $h$ are the azimuth and elevation respectively. An antenna efficiency factor of $\sim 65 \%$ has been measured, meaning a conversion factor of $1.25 \pm 0.09 \mathrm{~K} / \mathrm{kJy}$. Studies of neutral hydrogen in the disk of the Milky Way [4], of multipathing effects causing correlations between the periods of apparent solar oscillations observed by distant radio telescopes [5] and of polarized solar flares [6] have been published earlier.

The observations reported here have been made in two successive campaigns using different strategies, drift-scans and beam-switching. In both cases the telescope was pointed to fixed positions at which data were collected during fixed time intervals (40 min for drift-scans, $8 \mathrm{~min}$ for beam-switching) in a succession of $\sim 8 \mathrm{~s}$ integrations, each providing a frequency spectrum. We refer to a set of data collected when the telescope is pointing to a fixed position as a "pointing" and to each integration, or frequency spectrum, as a "measurement".

In what is referred as drift scans, the telescope was pointed to fixed positions on the Moon trajectory and data were collected during time intervals centred on the transit of the Moon at each of these positions. The time intervals were long enough (40 min) for the Moon to be off the telescope beam at the beginning and at the end of each time interval, the Moon being detected by subtraction of the power collected near the limits of the time interval from that collected at mid-interval.

In what is referred as beam-switching observations, much shorter time intervals were used $(8 \mathrm{~min})$, such as when the telescope was pointed to the Moon, the power collected from the Moon was essentially constant, the beam being much broader than the angle spanned by the Moon during the time interval. In this case, the empty sky contribution was subtracted by pointing the telescope off the Moon trajectory. Precisely, the telescope was pointed alternately on and off the Moon, off-the-Moon pointings alternating between $7^{\circ}$ up and $7^{\circ}$ down in elevation with respect to the Moon trajectory. Each pointing was such that the Moon would cross its azimuth at mid-period. The sequence of observations was: $8 \mathrm{~min}$ on, $8 \mathrm{~min}$ up, $8 \mathrm{~min}$ on, $8 \mathrm{~min}$ down, $8 \mathrm{~min}$ on, $8 \mathrm{~min}$ up, $8 \mathrm{~min}$ on, etc. It takes only $20 \mathrm{~s}$ to move the telescope from an "on" position to an "off" position and time intervals shorter than 8 min could have been chosen without much affecting the duty cycle. The choice of 8 min was in fact dictated by the concern of not overstraining the steering mechanism.

Calibrations using a noise source at the apex of the antenna, successively enabled and disabled for $\sim 1 \mathrm{~s}$, were performed at the beginning of each drift-scan, namely every $40 \mathrm{~min}$, but only once a day in the case of beam-switching observations. In many respects, the beam-switching campaign served as a prelude to the drift-scan campaign, the lessons being learned from it having been used to improve the quality of the latter. Among these was the choice of frequency, centred on the HI line in the beam-switching observations and shifted away from it, at a place where the system temperature is significantly lower, in the drift-scan campaign. We nevertheless present the results of both campaigns in what follows. 


\section{BEAM-SWITCHING OBSERVATIONS}

A first series of 14 beam-switching observations was performed between May $6^{\text {th }}$ and June $17^{\text {th }}, 2014$. Each observation, from Moon rise to Moon set, was made of successive "on" and "off" pointings, each lasting $\sim 8$ min, namely including nearly 60 frequency spectra (60 "measurements").

The strategy used to analyse the data is to consider pairs of successive "on-off" observations and to compare the associated antenna temperatures. In $8 \mathrm{~min}$, the Moon spans typically $2^{\circ}$, meaning $\pm 1^{\circ}$ with respect to the antenna pointing direction, small enough compared with the $\sigma$ of the beam $\left(2.3^{\circ}\right)$ to have a nearly negligible effect (it decreases the flux by only $3 \%$ ). On the contrary, the $7^{\circ}$ shift in elevation keeps the Moon contribution at a low enough level (between $1 \%$ and 3\%). The central frequency was fixed at $1420.4 \mathrm{MHz}$.

In order to eliminate contributions from HI clouds and RFIs, the measurement of the continuum antenna temperature is limited to a pair of intervals of frequency bins, below and above the $21 \mathrm{~cm}$ hydrogen line. A first linear fit is made over these intervals and measurements deviating from it by $\delta>10 \mathrm{~K}$ in absolute value (Figure $1 \mathrm{left}$ ) are rejected. The power spectrum is known to be well described by a linear form, with a slope of purely instrumental origin that depends on central frequency [3]. The fit is then repeated giving, for each spectrum $j$, values $a_{j}, b_{j}$ and $\chi_{j}^{2}$, of respectively the slope of the antenna temperature (in Kelvin per frequency bin), the antenna temperature at central frequency, and a measure of the quality of the fit (calculated for an arbitrary $1 \mathrm{~K}$ uncertainty and divided by the number of degrees of freedom). Figure 1 displays the distributions of $\delta, a_{j} / b_{j}$ and $\chi_{j}^{2}$. The inequalities $\left|a_{j} / b_{j}+0.45 \times 10^{-3}\right|<0.2 \times 10^{-3}$ and $\chi_{j}^{2}<28$ were required to be obeyed for the measurement to be retained. The distribution of $\chi_{j}^{2}$ indicates that a typical uncertainty of $4 \mathrm{~K}$ is attached to each frequency bin. Moreover, in order to exclude measurements where the system temperature and/or the sky antenna temperature are too far from average (possibly because of the proximity of the Sun, or of the Milky Way, or simply of ground) the inequality $205 \mathrm{~K}<b_{j}<255 \mathrm{~K}$ has also to be obeyed.

The next step checks the consistency of the sixty or so measurements of a same pointing. A linear fit of the form $b_{j}=b_{\text {mean }}+b^{\prime}\left(t_{j}-t_{\text {mean }}\right)$ is performed for each pointing, $t_{\text {mean }}$ being the time when the Moon crosses the meridian to which the telescope is pointing and $t_{j}$ the time of measurement $j$. Here again, the fit is made in two steps, the first step calculating the value of $b_{\text {mean }}$ and $b^{\prime}$ for the current pointing and the second step retaining only values of $b_{j}$ deviating from the linear fit by $\Delta b<1.5 \mathrm{~K}$ in absolute value (Figure 2 left). For a pointing to be retained in the final sample, it has to include a number $N>40$ of "good" measurements obeying this cut on $\Delta b$, to have a $\chi^{2}$ per degree of freedom not exceeding 0.75 and a value of $b^{\prime}$ not exceeding $10 \mathrm{mK}$ per time bin of $8.2 \mathrm{~s}$ in absolute value. Distributions of these quantities are displayed in Figure 2.

The above selection retains 77 pairs of "on-off" pointings with two values of $b_{\text {mean }}, b_{\text {on }}$ and $b_{o f f}$ measuring respectively the antenna temperatures of the Moon+sky and of the empty sky. If $b^{\prime}$ would cancel, namely if the empty sky antenna temperature were constant on a time scale of a pointing, the Moon antenna temperature would be obtained by simply subtracting $b_{o f f}$ from $b_{o n}$. But, as $b^{\prime}$ does not cancel, we need to correct for the evolution of the empty sky antenna temperature between the "on" and "off" pointings. As they are separated by $\sim 60$ time bins, the "on" pointing preceding the "off" pointing, we define the Moon antenna temperature as $A_{\text {Moon }}=b_{\text {on }}-\left(b_{o f f}-60 b^{\prime}\right)$. Its distribution is displayed in Figure 3. It has mean and rms values 

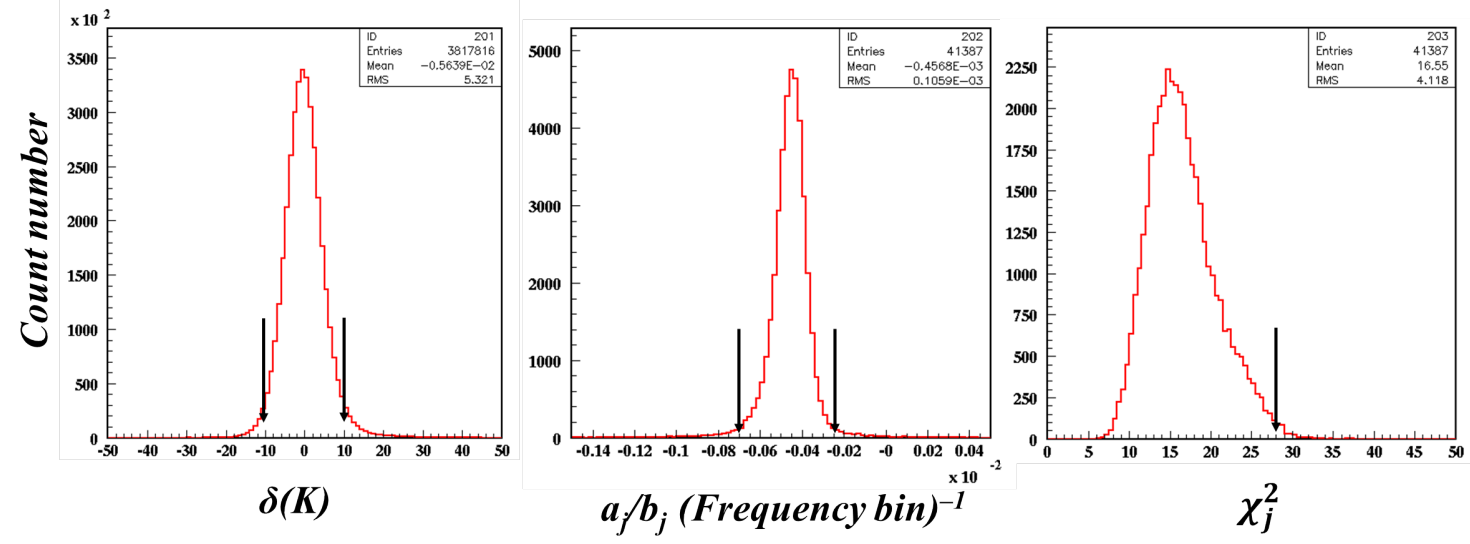

Fig. 1. Dependence of the antenna temperature on frequency: distributions of the deviation $\delta(\mathrm{K})$ of the measured antenna temperature from the linear fit; of the relative slope $a_{j} / b_{j}$ obtained from the linear fit (per frequency bin) and of $\chi_{j}^{2}$, the chi squared per degree of freedom describing the quality of the fit (see text). The arrows indicate the cuts that are applied.
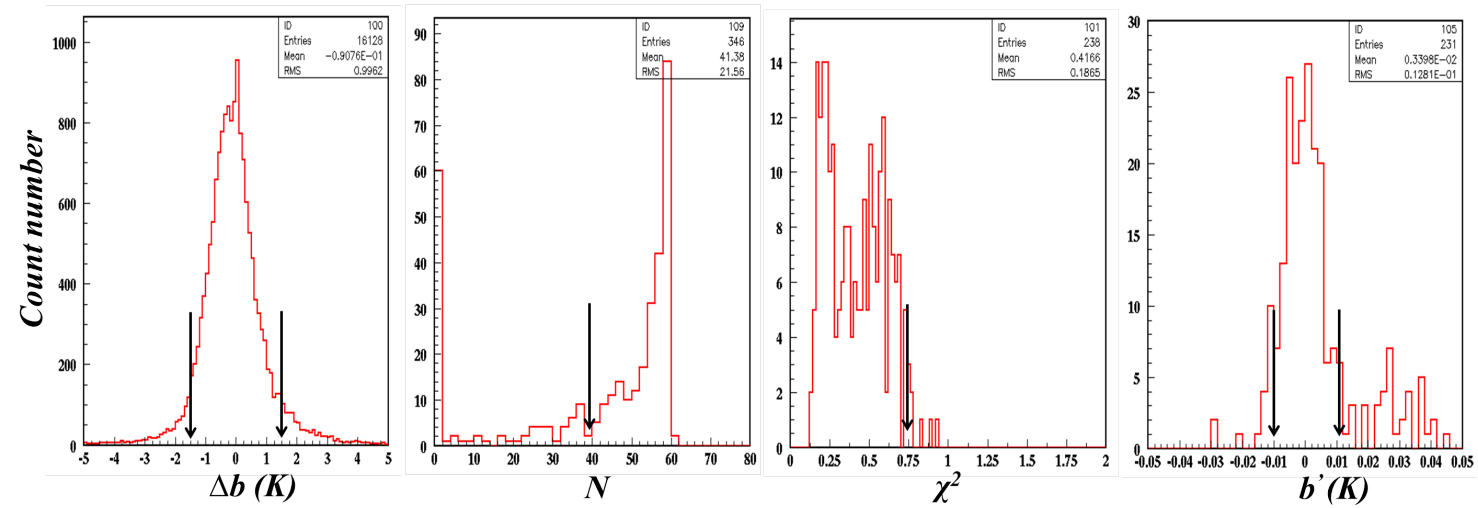

Fig. 2. Comparing antenna temperatures measured in the different frequency spectra of a same pointing: distributions of $\Delta b(\mathrm{~K})$, the deviation from the linear fit of the mean antenna temperature measured for each frequency spectrum of a same pointing (see text); of $N$, the number of measurements (frequency spectra) in each pointing obeying $\Delta b<$ $1.5 \mathrm{~K}$; of the value of $\chi^{2}$ describing the quality of the linear fit (see text); and of $b^{\prime}$ (in units of $\mathrm{K}$ per time bin of $\sim 8.2 \mathrm{~s}$ ). The arrows indicate the cuts that are applied.

of $0.6 \mathrm{~K}$ and $1.2 \mathrm{~K}$ respectively. Requiring the antenna temperature of the empty sky not to exceed $245 \mathrm{~K}(240 \mathrm{~K})$ reduces the sample to 57 (26) pairs of pointings and increases the mean value of the Moon antenna temperature to $0.7 \mathrm{~K}(0.9 \mathrm{~K})$. This illustrates the importance of systematic errors in this set of data. The result varies typically between $0.5 \mathrm{~K}$ and $0.9 \mathrm{~K}$ when the selection cuts are varied within reasonable limits, making a realistic evaluation of the final uncertainty difficult. Different analyses using triplets of successive pointings of the type "off-on-off" or "on-off-on" as 


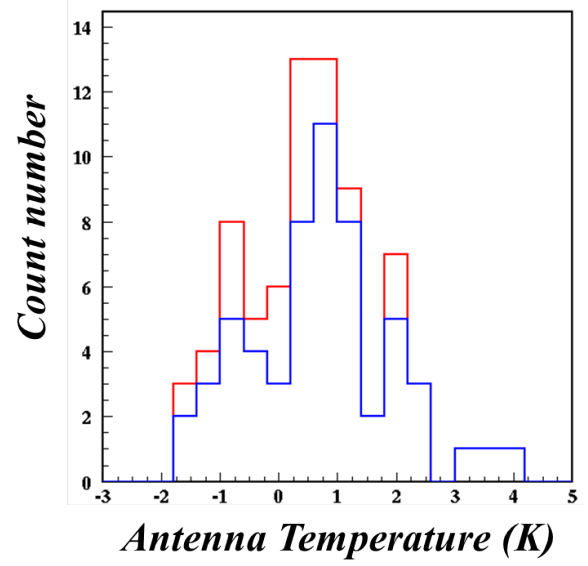

Fig. 3. Distribution of the antenna temperature of the Moon for the sample of 77 retained pairs of pointings (red) and for those obeying in addition the constraint $b_{\text {off }}<245 \mathrm{~K}$ (blue).

well as quintets of the type "on-up-on-down-on" have been made but failed to bring a significant improvement of the reliability and accuracy of the measurement. The main reason is the important variation of the empty sky temperature from one pointing to the next. The $b^{\prime}$ cut of $10 \mathrm{mK}$ per measurement means a maximal excursion of $\pm 0.3 \mathrm{~K}$ across the pointing and $\pm 0.6 \mathrm{~K}$ between pointings, commensurate with the Moon antenna temperature being measured. More frequent beam-switching would have improved the situation in this respect. Another lesson of these observations is the importance of choosing a frequency as free as possible of HI clouds and RFIs. In this respect, the choice of $1420.4 \mathrm{MHz}$ as central frequency was not optimal, the effective system temperature being higher there than at lower frequencies. We retain as final result a Moon antenna temperature of $0.7 \pm 0.2 \mathrm{~K}$, dominated by systematic uncertainties.

\section{DRIFT SCANS}

Taking advantage of the experience gained with the series of beam-switching observations, a second campaign was made, this time collecting a large number of drift scans, each lasting 40 min, such that the telescope be pointing to the Moon at mid scan, namely 20 min after start. As 20 min corresponds to an angular drift of $5^{\circ} \cos \delta$, where $\delta$ is the declination of the Moon, and the antenna lobe has a $\sigma$ of $2.3^{\circ}$, the sagitta of the dependence of the detected flux on time is more than $1-\exp \left[-\frac{1}{2}(5 / 2.3)^{2}\right] \sim 90 \%$ of the true flux. As we are close to a minor lunar standstill, the Moon declination remains small and the $\cos \delta$ factor has little influence. The frequency was fixed at $1417.6 \mathrm{MHz}$ in order to be as free as possible from RFIs and HI clouds and observations were performed between July $16^{\text {th }}$ and October $12^{\text {th }}, 2014$. After having rejected scans pointing too close to the Sun, the Milky Way or obstacles on ground, scans where the Moon at mid-scan is more than $0.8^{\circ}$ away from the pointing direction of the telescope and scans displaying sudden changes of gain resulting from strong RFIs, we are left with a total of $80 \mathrm{drift}$ scans (pointings) to be analysed. 
In a first phase, we fit a straight line, $a i+b$, to the antenna temperature $T_{i}$ measured in bin $i$ of each frequency spectrum (of which there are 310 per drift scan). The resulting distributions of $a, b, a / b, \chi^{2}$ and $\left|\delta_{i}\right|=\left|T_{i}-a i-b\right| / \Delta_{i}$ (using an uncertainty $\Delta_{j}=2 \mathrm{~K}$ ) are displayed in Figure 4. We only retain measurements having $\chi^{2}<5$ units per degree of freedom (of which there are 138), $-9.4 \times 10^{-4}<a / b<-5.4 \times 10^{-4},-0.22<a<-0.12$ and $b<300 \mathrm{~K}$. Here, $a$ is in K per frequency bin and $a / b$ in (frequency bin) ${ }^{-1}$. We note that the mean value of $a / b,-7.4 \times 10^{-4}$, and that found in the beam-switching data, $-4.5 \times 10^{-4}$, are consistent with the known frequency dependence [3] of $\sim 80 \mathrm{ppm} / \mathrm{MHz}$. We then repeat the fit retaining only frequency channels having $\left|\delta_{i}\right|<7 \mathrm{~K}$ and calculate the new values of the preceding quantities, which are also displayed in Figure 4 together with the earlier values. Finally, we only retain drift scans having a number $N$ of retained measurements in excess of 270 (Figure 4). There are 64 of them.

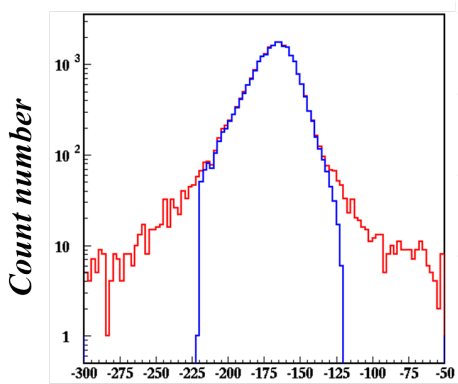

a (\%)

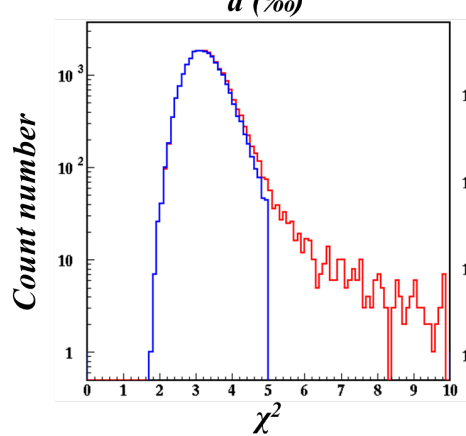

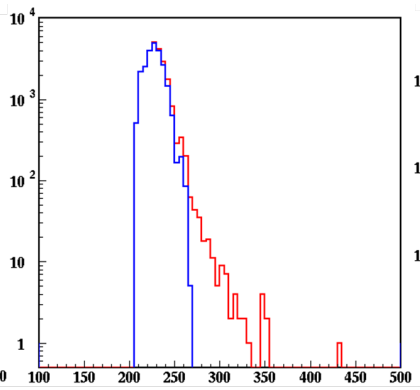

$\boldsymbol{b}(\boldsymbol{K})$

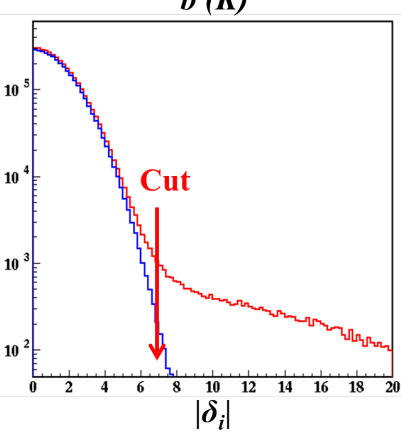

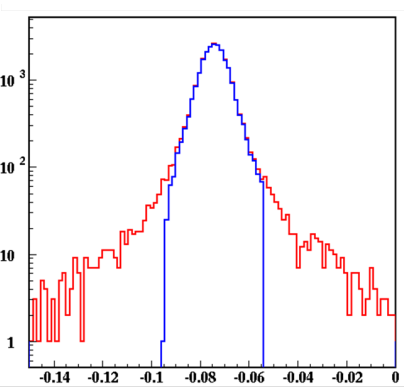

$a / b(\%)$

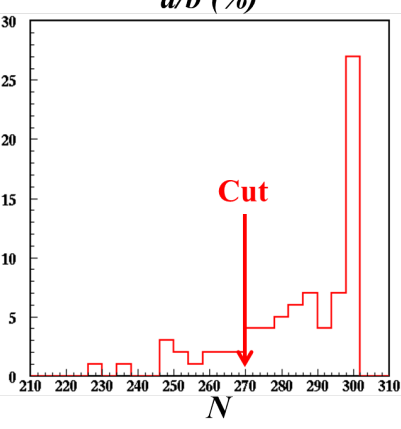

Fig. 4. From left to right and top to bottom: distributions of $a(\%), b(\mathrm{~K}), a / b(\%), \chi^{2}$ and $\left|\delta_{i}\right|$ before (red) and after (blue) selection of the retained measurements. In the first four panels there is one entry per measurement, namely per frequency spectrum. In the fifth panel, there is one entry per frequency bin. The last panel displays the distribution of the number $N$ of retained measurements per drift scan (one entry per drift scan). Only drift scans having $N>270$ are retained for further analysis.

For each of the 64 retained drift scans, the $b_{j}$ values of each retained measurement $j$ (of which there are $N>270$ per drift scan) are fitted to a form

$b_{j}=T_{\text {sky }}[1+\xi(j-159)]+T_{\text {Moon }} \exp \left[-\frac{1}{2}(j-159)^{2} \cos ^{2} \delta / 72.4^{2}\right]$.

Here, the first term describes an empty sky contribution that varies linearly with time, with mean value $T_{\text {sky }}$ and relative slope $\xi$. The second term describes the contribution $T_{M o o n}$ of the Moon, modulated by the beam as the Moon drifts across it. The values 159 and 72.4 correspond 
respectively to the value of $j$ for the measurement pointing to the Moon and to the lobe width $\left(\sigma=2.3^{\circ}\right.$, meaning $9.2 \mathrm{~min}$ or $72.4 j$ bins divided by $\cos \delta$ ). The resulting $\chi^{2}$ distribution (calculated per degree of freedom for an uncertainty of $1 \mathrm{~K}$ on $b_{j}$ ) is displayed in Figure 5 (left). The fit ignores the first four measurements $(j<5)$ because they are used for calibration. Retaining only scans having $\chi^{2}<2$, we are left with a final sample of 52 drift scans. The distribution of the measured antenna temperature, from which the fitted sky temperature $T_{s k y}[1+\xi(j-159)]$ has been subtracted, is compared in Figure 5 (right) with the result of the fit.
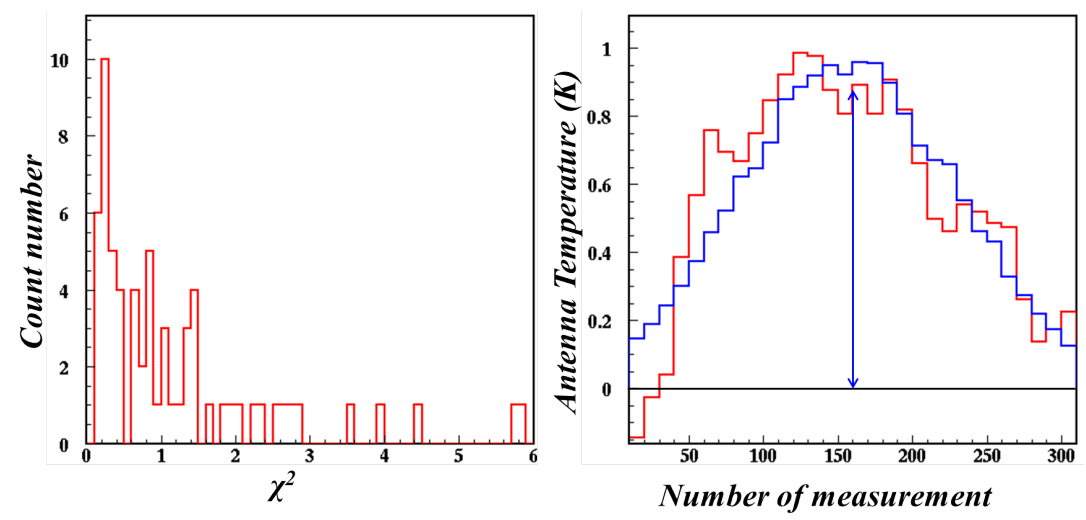

Fig. 5. Left: Distribution of the $\chi^{2}$ per degree of freedom obtained for the 64 final drift scans from fits of the $b_{j}$ values to a form $T_{s k y}[1+\xi(j-159)]+T_{M o o n} \exp \left[-\frac{1}{2}(j-159)^{2} / 72.4^{2}\right]$. Right: Evolution of the antenna temperature as a function of measurement number, measured (red) and fitted (blue), from which the fitted empty sky temperature has been subtracted.
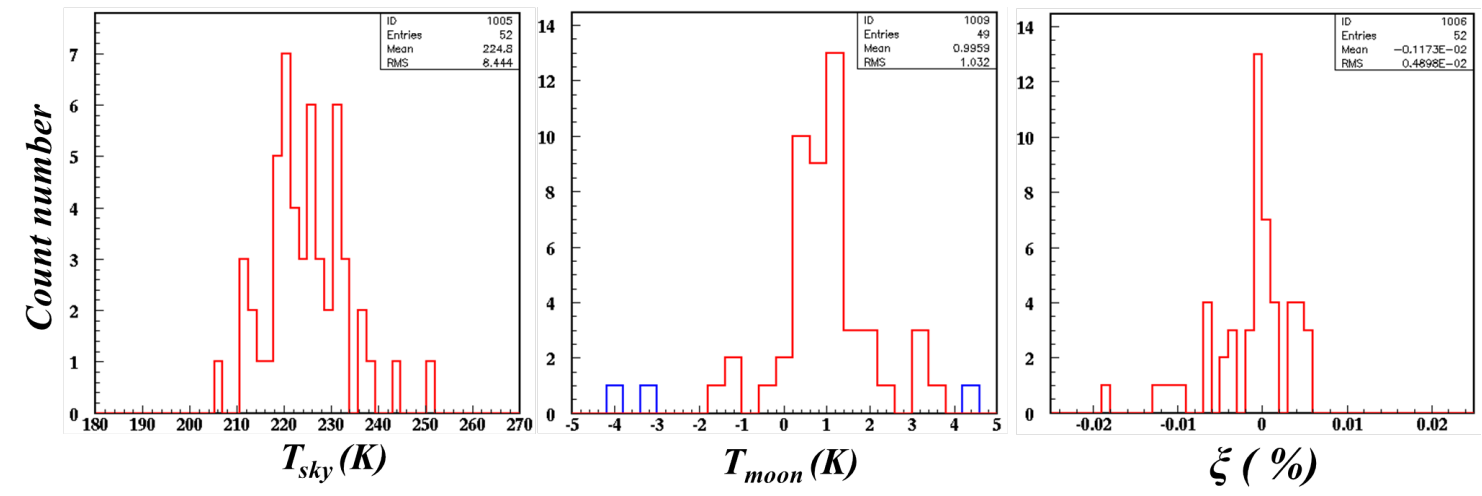

Fig. 6. Left: Mean sky temperature, $T_{s k y}(\mathrm{~K})$. Centre: Moon temperature, $T_{M o o n}(\mathrm{~K})$. Right: Time slope of the sky temperature, $\xi$ (in \%).

Distributions of $T_{s k y}, T_{M o o n}$ and $\xi$ are displayed in Fig. 6. The dispersion of $\xi \sim 5 \times 10^{-5}$, means $10 \mathrm{mK}$ per measurement, comparable with the value found for the beam-switching observations. However, in the present case, there is a calibration at the beginning of each drift scan, the 
telescope stands still during the whole scan and, data being collected continuously, the variation of the empty sky temperature is kept under good control. The value of $T_{M o o n}$, excluding 3 drift scans where it deviates by more than $3 \mathrm{~K}$ from its mean, has a mean value of $1.00 \mathrm{~K}$ with an rms value of $1.03 \mathrm{~K}$. Ignoring possible systematic errors would mean an uncertainty of $1.00 / \sqrt{4} 9=0.14 \mathrm{~K}$ on $T_{M o o n}$. The mean sky temperature has a mean value of $225 \mathrm{~K}$ with an rms value of $8 \mathrm{~K}$ and its time slope is centred on $-12 \mathrm{ppm}$ with an rms value of $49 \mathrm{ppm}$ per $8.2 \mathrm{~s}$, meaning respectively $\sim-0.3 \%$ and $\sim 1.4 \%$ over a full scan.

\section{DISCUSSION}

We have obtained two different but consistent evaluations of the Moon antenna temperature, $0.7 \pm 0.2 \mathrm{~K}$ and $1.00 \pm 0.14 \mathrm{~K}$. However, because of the different choices of frequency and of the better control over systematic errors offered by the drift scans, we prefer to retain the latter as our final result, using the former as a simple consistency check. Indeed, the robustness of the results with respect to changes in the selection criteria, an indicator of the importance of systematic uncertainties, is much better in the drift-scan campaign than in the beam-switching campaign.

To the measured value of $T_{M o o n}, 1.00 \pm 0.14 \mathrm{~K}$, we must add the contribution of the empty sky hidden behind the Moon and to its uncertainty a possible systematic contribution. The fraction of the solid angle, weighted by the gain of the antenna, covered by the Moon (angular diameter of $31^{\prime}$ and lobe $\sigma$ of $2.3^{\circ}$ ) is $0.66 \%$. The antenna temperature measured on the empty sky takes values between $200 \mathrm{~K}$ and $250 \mathrm{~K}, \sim 225 \mathrm{~K}$ on average. Most of it is an effective system temperature, including all contributions other than from astronomical origin, the real contribution of the empty sky that is hidden behind the Moon is nearly negligible in comparison, just a few $\mathrm{K}$ (the HI line is out of the bandwidth). For an estimated empty sky antenna temperature of $5 \mathrm{~K}$, we must add 0.03 $\mathrm{K}$ to the Moon antenna temperature.

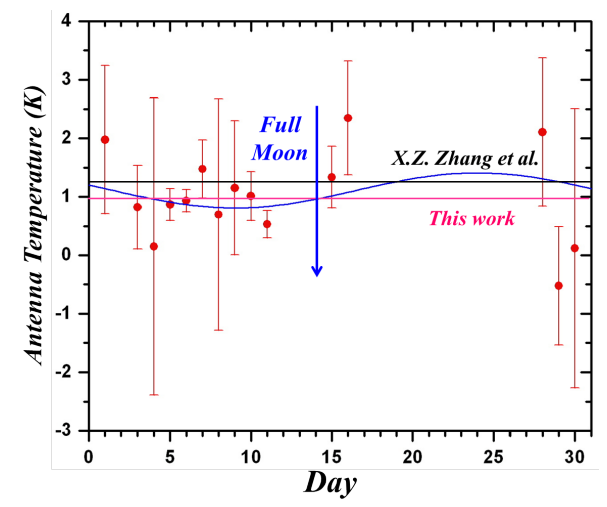

Fig. 7. Variation of the measured antenna temperature of the Moon (red) as a function of its phase $\varphi$ (measured in days from 0 to 30 starting at New Moon) together with the result of a fit (blue) to a form $T_{0}+T_{1} \cos \left(\varphi+\varphi_{0}\right)$, with $T_{0}=1.11 \mathrm{~K}, T_{1}=0.30 \mathrm{~K}$ and $\varphi_{0}=-6.5^{\circ}$. The present result of $T_{M o o n}=1.03 \mathrm{~K}$ (no phase dependence) is shown as a magenta line and that of Zhang et al. as a black line.

From the robustness of this result as a function of the values adopted for the cuts, we estimate a systematic uncertainty of $\sim 0.15 \mathrm{~K}$. Adding it in quadrature to the value obtained from the 
rms dispersion of $T_{M o o n}$ around its mean, we retain as final result for the Moon antenna temperature: $T_{M o o n}=1.03 \pm 0.20 \mathrm{~K}$. An estimate of the ultimate sensitivity of the instrument, as defined at the level of two standard deviations, is $\sim 0.4 \mathrm{~K}$ for the antenna temperature, meaning $\sim 300$ Jy for the flux density, in agreement with earlier coarser estimates. The main factors limiting the sensitivity of the instrument are not electronic noise but time variations of the empty sky temperature, due in part to variations of the gain, probably associated with its dependence on ambient temperature, and the presence of "bad" measurements, associated in part with RFIs. Operating the telescope in a quieter environment and controlling the temperature would undoubtedly improve its performance and probably lower its sensitivity limit down to noise level.

The Moon brightness temperature averaged over the whole disk has recently been measured [7] at $1420 \mathrm{MHz}$. The result, $233 \mathrm{~K}$, is in good agreement with previous observations. The measurement was done on January $7^{\text {th }}$ and $8^{\text {th }}, 2009$, respectively 3 and 2 days before full Moon. As the Moon angular radius is $31^{\prime}=4.5 \times 10^{-3} \mathrm{rad}$, the Moon solid angle is $\Omega=$ $\pi 4.5^{2} \times 10^{-6}=63.6 \times 10^{-6} \mathrm{sr}$ and the flux density is therefore $2 k_{B} \Omega \lambda^{-2} \times 233=233 \times 0.0636 \times$ $10^{-3} \times(0.21)^{-2} \times 2 \times 1.38 \times 10^{-23} \times 10^{26}=0.93 \mathrm{kJy}$, corresponding to an antenna temperature of $1.16 \mathrm{~K}$ for the VATLY radio telescope in very good agreement with our measurement. Conversely, our result means a flux density of $0.83 \pm 0.16 \mathrm{kJy}$ and a black body temperature of $207 \pm 40 \mathrm{~K}$.

Figure 7 displays the variation of the Moon antenna temperature as a function of the phase $\varphi$ of the Moon together with the result of a fit to a form $T_{0}+T_{1} \cos \left(\varphi+\varphi_{0}\right)$. Including such a phase dependence causes the $\chi^{2}$ to decrease from 11.7 for 14 degrees of freedom to 10.2 for 12 degrees of freedom, implying that there is no evidence for any dependence of the Moon temperature on the phase. Indeed, it is well established [1] that the Moon brightness temperature at $1.4 \mathrm{GHz}$ varies by less than $1 \%$ over a lunar month.

\section{ACKNOWLEDGMENT}

We are deeply indebted to Dr A. Rogers for his interest and helpful comments and to the anonymous referee for several suggestions that greatly helped improving the quality of the manuscript. Financial support is acknowledged from the Institute for Nuclear Science and Technology (VINATOM/MOST), the Vietnam National Foundation for Science and Technology Development (NAFOSTED) under grant number 103.08-2012.34, the World Laboratory, the Odon Vallet Foundation and the Rencontres du Viet Nam.

\section{REFERENCES}

[1] P. G. Mezger and H. Strassl, Planet. Space Sci., 1 (1959) 213.

J. E. Baldwin, MNRAS 122 (1961) 513.

C.E. Heiles and F.D. Drake, Icarus 2 (1963) 281.

V. A. Razin and V. T. Fedorev, Radiofiz. 6 (1963) 1052.

P. H. Moffat, MNRAS 160 (1972) 139.

F. P. Schloerb, D. O. Muhleman and G. L. Berge, Icarus 29 (1976) 329.

[2] N. V. Hiep et al., The VATLY radio telescope, Comm. Phys. 22(4) (2012) 365;

See also:

N. V. Hiep (on behalf of VATLY), Presented at the Second Academic Conference on Natural Science for Master and $\mathrm{PhD}$ students from Cambodia, Laos, Malaysia and Vietnam, 11-15 October 2011, Vinh, Vietnam.

P. T. Anh (on behalf of VATLY) presented at the 7th Annual Conference of the Thai Physics Society, May 9-12, 2012, Phranakhon Si, Ayutthaya, Thailand. 
N. V. Hiep (on behalf of VATLY) presented at the 1st Southeast Asian Young Astronomers Collaboration (SEAYAC) Meeting, November 5-9, 2012, Puerto Princesa City, Palawan, Philippines.

[3] N. T. Phuong et al., The VATLY radio telescope: performance study, Comm. Phys Vietnam. Vol. 24, No. 3 (2014), pp. 257-266 DOI:10.15625/0868-3166/24/3/3981

[4] N. V. Hiep et al., 2013, Mapping atomic hydrogen in the disk of the Milky Way, Comm. Phys. Vietnam. Vol.23, No 2 (2013), 107-119.

N. V. Hiep, Observation of the $21 \mathrm{~cm}$ sky using the VATLY radio telescope, Master thesis, presented at Vietnam Institute of Physics, 2012.

[5] N.V. Hiep et al., Radio Observation of Solar-Activity-Related mHz Oscillations, Sol. Phys. 289(3) (2014) 939-950.

N. T. Phuong (on behalf of VATLY) presented at 2nd South East Asia Young Astronomer Colaboration, November 19-22, Bandung, Indonesia 2013.

P. T. Nhung (on behalf of VATLY) presented at the Conference "Windows on the Universe", August 11-17, Quy Nhon, Vietnam, 2013.

P. N. Diep et al., Correlated Oscillations Due to Similar Multipath Effects Seen in Two Widely Separated Radio Telescopes, Publications of the Astronomical Society of Australia, 31, e029 doi:10.1017/pasa.2014.24.

N. T. Phuong (on behalf of VATLY), presented at Workshop on Astronomy and Astrophysics in Vietnam, April 7-11, Institute for Nuclear Science and Technology, Hanoi, Vietnam.

P. N. Diep (on behalf of VATLY), presented at 12th Asia-Pacific Region IAU Meeting, Daejeon, Korea, 2014.

[6] N.T. Thao et al., Evidence for polarized solar flare emission from simultaneous radio observations from Australia and Vietnam, submitted for publication in Comm. Phys., 2014.

N. T. Phuong, Solar and other observations using VATLY radio telescope, Master thesis, in preparation.

B. V. Tuan, A study of the six major solar flares recently detected from Ha Noi and Learmonth at $1415 \mathrm{MHz}$ compared with other frequencies, internship report presented at University of Science and Technology of Hanoi, Master Space and Applications, September, 2014.

N. H. Phuong Thanh, A study of 34 solar flares detected by Hanoi and Learmonth telescope at $1415 \mathrm{MHz}$ between 2012 and 2014, internship report presented at University of Science and Technology of Hanoi, Master Space and Applications, September, 2014.

[7] Xi-Zhen Zhang, A. Gray, Yan Su, Jun-Duo Li, T. Landecker, Hong-Bo Zhang and Chun-Lai Li, 2012, New radio observations of the Moon at L band, RAA 12/9, 1297. 